\title{
INTEGRATED COMMUNICATIONS AND OPTICAL NAVIGATION SYSTEM
}

\author{
J. Mueller, G. Pajer, and M. Paluszek \\ Princeton Satellite Systems \\ 6 Market Street, Suite 926, Plainsboro, NJ 08536, USA
}

\begin{abstract}
The Integrated Communications and Optical Navigation System (ICONS) is a flexible navigation system for spacecraft that does not require global positioning system (GPS) measurements. The navigation solution is computed using an Unscented Kalman Filter (UKF) that can accept any combination of range, range-rate, planet chord width, landmark, and angle measurements using any celestial object. Both absolute and relative orbit determination is supported. The UKF employs a full nonlinear dynamical model of the orbit including gravity models and disturbance models. The ICONS package also includes attitude determination algorithms using the UKF algorithm with the Inertial Measurement Unit (IMU). The IMU is used as the dynamical base for the attitude determination algorithms. This makes the sensor a more capable plug-in replacement for a star tracker, thus reducing the integration and test cost of adding this sensor to a spacecraft. Recent additions include an integrated optical communications system which adds communications, and integrated range and range rate measurement and timing. The paper includes test results from trajectories based on the NASA New Horizons spacecraft.
\end{abstract}

\section{NOMENCLATURE}

a planet radius or half distance between landmarks

$f$ focal length

$k_{1} \quad$ first-order optical distortion coefficient in the imager focal plane

$k_{2} \quad$ second-order distortion coefficient in the imager focal plane

$l_{i} \quad$ vector from the Sun to the planet center or the center of a straight line joining two landmarks

$o_{x} \quad$ image center $x$ coordinate

$o_{y}$ image center $y$ coordinate

$p_{c}$ the position in the camera frame

$p_{w}$ the position in the world frame

$R$ transforms from the world to the camera frame 
$r$ the radial distance in the imager focal plane

$r_{d}$ radial distance in the pixel frame

$s_{x} \quad$ pixel size in the $x$ direction

$s_{y}$ pixel size in the $y$ direction

$T$ camera offset from the origin of the world coordinates

$u$ the vector from the spacecraft to a reference star

$x$ undistorted position in the imager focal plane

$x_{d}$ distorted $x$ location in the imager focal plane

$x_{\mathrm{im}} \quad x$ location of the image in the focal plane

$y_{\mathrm{im}} y$ location of the image in the focal plane

$y$ undistorted position in the imager focal plane

$y_{d}$ distorted $y$ location in the imager focal plane

$z \quad z$-axis position

$\alpha$ pixel size ratio

$\theta_{1}$ the angle between two planets or two landmarks on two planets

$\theta_{2}$ the angle between two landmarks on a single planet or the chord width of the planet

$\theta_{3}$ the angle between a landmark vector and a star

$\rho$ range

\section{INTRODUCTION}

Spacecraft navigation has been done primarily by means of range and range rate measurements from ground based antennas. This has proven to be very successful and reliable. The basis of these measurements is that the range

$$
\rho=\sqrt{x^{2}+y^{2}+z^{2}}
$$

and the range rate

$$
\dot{\rho}=\frac{\vec{v}^{\mathrm{T}} \vec{r}}{\rho}
$$

contains information about all of the spacecraft states: the position in Earthcentered-inertial (ECI) reference frame, $\vec{r}=(x, y, z)$, and velocity $\vec{v}=\dot{\vec{r}}$. If the geometry changes sufficiently as measurements are taken, all six state variables can be recovered. Once the state is reasonably well known, a recursive estimator can track the state with single range and range rate measurements. However, since typically knowledge of the spacecraft state is only required for occasional course correcting maneuvers, a batch estimator on the ground is usually employed. When doing ground-based navigation, additional state variables, such as the latitude and longitude of the tracking antenna, are usually also estimated.

The major drawback with this approach is that it ties up ground antennas and facilities, causing it to be expensive. Furthermore, this method is not robust 
to loss of contact, space segment degradations, and other factors. Additionally, if frequent navigation solutions are required, it may not be possible to schedule the observations when needed. Some proposed missions, including solar sails, spacecraft with continuous propulsion, and formation flying missions, require frequent updates. During the terminal approach in a rendezvous, it may be necessary to update the navigation solution at a very high rate. For this reason, optical navigation has been proposed for spacecraft navigation.

Position can be determined from the angle between a star and the vector to a landmark. This approach was used on Apollo [1] as a backup to the conventional range and range rate measurements which used the Telemetry, Tracking, and Control system. The range and range rate measurements were obtained from the antennas on the ground.

The first major attempt to shift navigation from ground-based to onboard operations was made by NASA during the Deep Space 1 (DS-1) mission [2,3] in 1999. During this mission, autonomous navigation was operational during the cruise phase, until the failure of the onboard star tracker. The principle adopted by DS-1 for autonomous orbit determination was optical triangulation.

Reduced State Encounter Navigation (RSEN), using optical images as the sole data type, was used on DS-1 to track comet Borrelly for $2 \mathrm{~h}$ through the closest approach. Reduced state encounter navigation is initialized using the last ground or onboard estimate of spacecraft state relative to the target just before commencement of the final approach phase. Reduced state encounter navigation was also used in the Stardust mission during comet Wild-2 flyby and in the Deep Impact mission to navigate the impactor to hit an illuminated area on the comet Tempel 1 and by the flyby spacecraft to track the impact site.

The Japanese asteroid return mission Hayabusa [4,5] employed wide angle cameras for onboard navigation, in conjunction with Light Detecting and Ranging (LIDAR) for measurement of altitude with respect to the asteroid Itokawa. A target marker was dropped to act as a navigation aid by posing as an artificial landmark on the surface. A laser range finder was used to measure the altitude as the spacecraft got close (less than $120 \mathrm{~m}$ ) to the asteroid. Fan beam sensors detected potential obstacles that could hit the solar cell panels.

European Space Agency's Small Missions for Advanced Research in Technology (SMART-1) mission employed the Advanced Moon Imaging Experiment (AMIE) camera for Onboard Autonomous Navigation (OBAN) [6,7]. The Moon and Earth were used as beacons. The beacons were too bright to enable observation of stars with the AMIE camera. A star tracker was used to estimate pointing direction. The OBAN framework is also being used for designing a vision-based autonomous navigation system for future manned Mars exploration missions [8].

The Near-Earth Asteroid Rendezvous (NEAR) Shoemaker mission relied heavily on optical navigation [9]. The system located small craters on the surface of Eros and used them as references. This enabled rapid orbit determination near 
and around Eros. The more traditional approach of using the planet centroids was not feasible due to the irregular shape of Eros. The planet centroid technique was used on Mariner 9, Vikings 1 and 2, Voyagers 1 and 2, and Galileo. It was also used by NEAR on its approach to the asteroid Mathilde. More recently, this approach was used on the Mars Reconnaissance Orbiter (MRO).

Autotracking of landmarks is discussed further in [10]. This is a complex combination of premission identification of landmarks and autonomous landmark identification and tracking. Autotracking is desirable for planetary orbit operations or landings.

The examples cited above illustrate the utility of optical navigation to a wide range of missions. In this paper, a flexible ICONS for deep space operations is presented. The overall system design is first presented in section 2. Simulation results are presented in section 3 .

\section{OVERALL SYSTEM DESIGN}

\subsection{Integrated Communications and Optical Navigation System}

Deep space or geosynchronous satellites have an ICONS sensor which performs simultaneous optical navigation, optical communications, and attitude determination. Each sensor has two articulated telescopes with the entire sensor on a turntable. Each telescope has an imaging chip, laser, photodiode, and related optics and fixtures. The communication systems talk with reference satellites in Earth orbit. The reference satellites use GPS to compute their positions and velocity. Each reference spacecraft has an ICONS sensor for communicating with the deep space spacecraft or each other for cross links. Besides communications, the optical channel provides range and range rate measurements for the Earth reference.

The ICONS telescopes can be used simultaneously for communications and measuring the star field or planet references for navigation information. The use of multiple measurements provides higher accuracy. However, navigation does not rely on the communications channel and can operate indefinitely without range and range rate updates. The operation of the communication and navigation systems is discussed in the following subsections.

\subsection{Observables}

The optical navigation system is designed to use multiple measurement sources. These include: planet, moon or asteroid chordwidth, landmark size, centroid and star, landmark and star, landmark and landmark, landmark and centroid, star 
and star (for attitude determination). Figure 1 shows the geometry of optical navigation in two dimensions. The angles $\theta_{k}$ are three possible types of angle measurements. The angle between two planets is

$$
\begin{aligned}
\cos \theta_{1} & =\frac{\vec{\rho}_{1}^{\mathrm{T}} \vec{\rho}_{2}}{\left|\vec{\rho}_{1}\right|\left|\vec{\rho}_{2}\right|} \\
& =\frac{\left(\vec{r}+\vec{l}_{1}\right)^{\mathrm{T}}\left(\vec{r}+\vec{l}_{2}\right)}{\left|\left(\vec{r}+\vec{l}_{1}\right)\right|\left|\left(\vec{r}+\overrightarrow{l_{2}}\right)\right|}
\end{aligned}
$$

where $\vec{\rho}_{i}$ is the vector from the spacecraft to either the planet $i$ th landmark location or the planetary center; $\vec{l}_{i}$ is the vector from the Sun to either the planet's $i$ th landmark location or the planetary center; and $\vec{r}$ is the vector from the space-

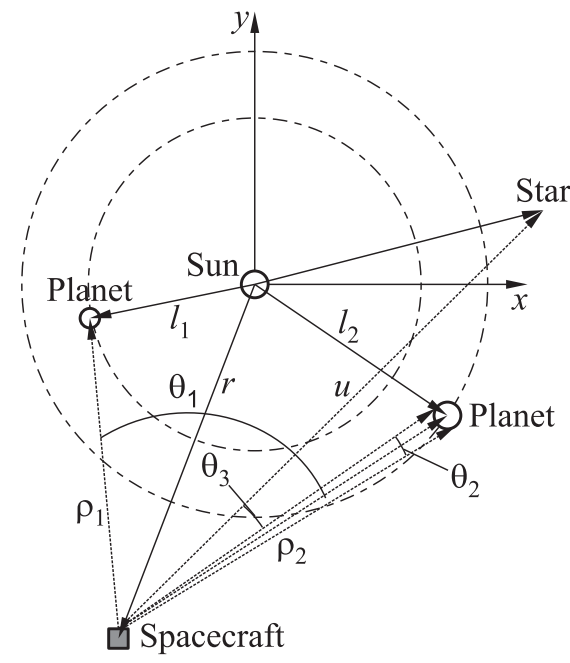

Figure 1 Measurement geometry craft to the Sun.

The angle between landmarks on a planet or the angle subtended by the planetary chord is defined by

$$
\sin \frac{\theta_{2}}{2}=\frac{a}{|\vec{l}-\vec{r}|}
$$

where $a$ is either the planet radius or half the distance between two landmarks, and $\vec{l}$ is the vector from the Sun to either the planet center or the center of the straight line joining the two landmarks.

The angle between a landmark vector and a star is defined by

$$
\cos \theta_{3}=\frac{\vec{u}^{\mathrm{T}}(\vec{r}-\vec{l})}{|\vec{r}-\vec{l}|}
$$

where $\vec{u}$ is the vector from the spacecraft to a reference star.

In order to compute the navigation solution, it is necessary to have at least one measurement of type $\theta_{1}$ or $\theta_{2}$ and two other measurements of any type. While these three measurements form the minimal required set, more measurements (whose statistics are known accurately) generally lead to better accuracy in the navigation solution.

Figure 2 shows the values of the observables for the New Horizons mission. The planet chords are the angular width for all planets. The power is the radiant flux received from each planet. The minimum range is the range to the closest 


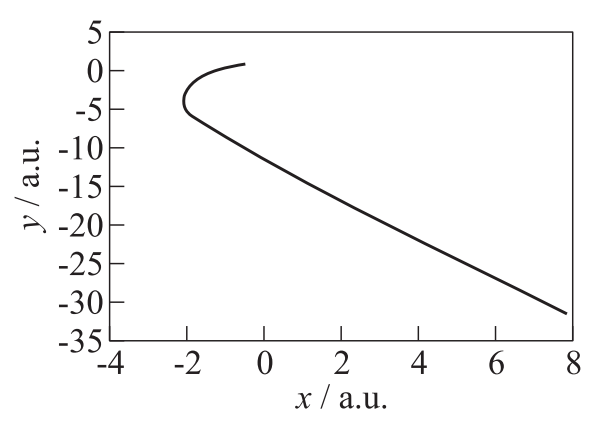

(a)

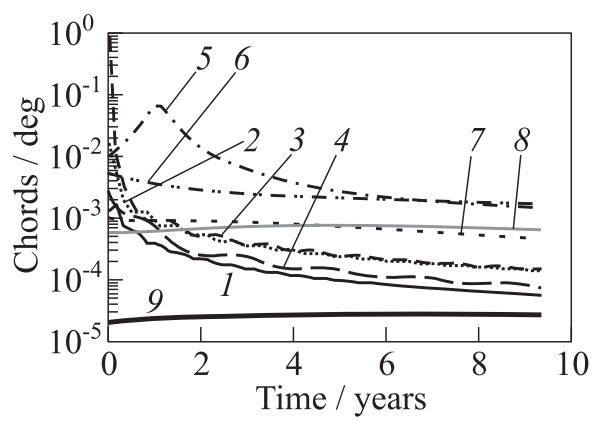

(b)

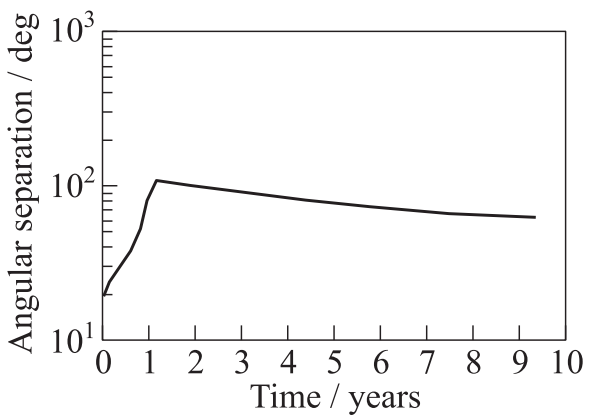

(c)

Figure 2 New Horizons mission: (a) spacecraft position; (b) planet chords (1 Mercury; 2 - Venus; 3 - Earth; 4 - Mars; 5 - Jupiter; 6 - Saturn; 7 - Uranus; 8 - Neptune; and 9 - Pluto); and (c) planet angular separation

planet and the minimum angular separation is the minimum separation among all pairs of planets.

The ICONS can always measure angles between planetary pairs since its telescopes are articulated. The angle between the telescopes is measured using stellar attitude determination of both telescopes. It will rarely be the case that two planets will appear in a single telescope's field-of-view.

\subsection{System Design}

The ICONS is a two telescope navigation and attitude determination system (Fig. 3). The design includes refracting telescopes with a 50-millimeter focal length and 25-millimeter aperture each with a semiconductor laser, photodiode, and frequency selective beam splitter. Each telescope has an azimuth and elevation gimbal and both telescopes are mounted on a turntable. A calibration 


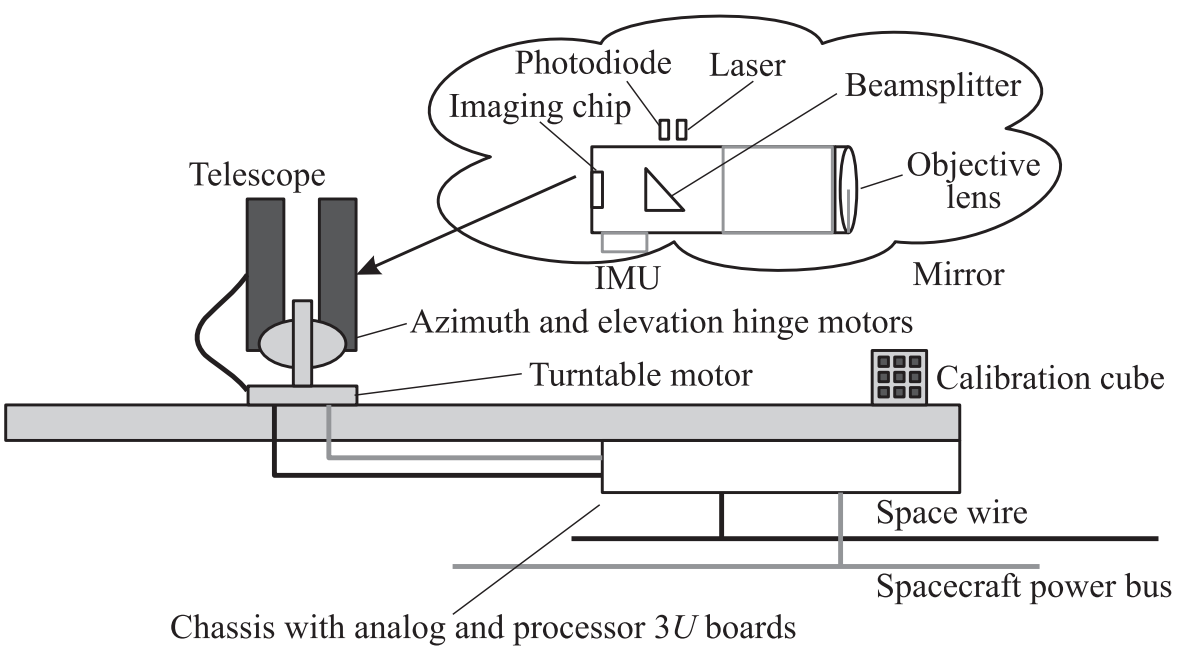

Figure 3 Sensor design. This diagram shows a beam splitter for the laser that may be eliminated if the laser frequency is outside of the bandwidth of the imaging chip

cube is included with precision LED (light eminent diode) sources for imager and optical path calibration. Built-in MEMS (mircoelectromechanical systems) IMU with accelerometers and gyroscopes are mounted to each telescope. Ultrasonic motors are used for the gimbal and turntables that have angle encoders to measure angles to the tolerance required by attitude determination.

\subsection{Optical Communication}

The optical communication subsystem consists of a modulated laser source, beam shaping optics, beam steering optics, and a receiver. The receiver shares the same field of view as the navigation system, so some means is needed to discriminate the communication signal from radiation received from navigation objects. This can be accomplished by choosing a wavelength for communication to which the navigation imager is insensitive, or by selectively redirecting the laser energy away from the imaging optics and into the communication receiver.

It is, perhaps, easier to implement the former case by choosing a communication wavelength in the infrared, beyond the cutoff wavelength of the imager. Redirecting the energy is possible by the use of wavelength notch beamsplitter, but care must be taken to avoid scattering of the laser light, and the extinction of the beam splitter must be adequate to prevent leakage of light on to the focal plane array. Both of these problems can be avoided by selecting a wavelength outside the sensitive band of the detector. 
The simplest laser system to use would be a semiconductor diode laser. Many such lasers can be electronically modulated, and there is minimal ancillary equipment needed for operation. If power in excess of a few hundred watts is needed, a diode-pumped Nd:YAG laser may be a suitable choice. Beam quality from a diode laser is rather poor; so, it would be necessary to use beam shaping optics to bring the beam closer to the ideal Gaussian profile while simultaneously matching the numerical aperture of the objective lens.

The receiver uses the same objective lens and beam steering optics as the transmitter. The collected light is focused onto a single channel optical detector. In many cases of interest, the received optical flux will be very low and photon counting techniques will be needed. In these cases, the detector can be either an avalanche photodiode (APD) or a photomultiplier tube (PMT). For shorter distance communication, a pin photodiode operating as a current source would serve adequately.

The primary limitation on performance, aside from power reduction due to beam spreading over distance, is signal fading due to vibrational jitter in the transmitter system. Low-frequency jitter can be reduced by active attitude control of the satellite. The effect of high-frequency jitter can be mitigated by intentionally degrading the beam so that the beam spreads over an angle that is 6 to 10 times the expected angular jitter. The trade-off for this procedure is, of course, reduced intensity at the receiver.

\subsection{Software}

\subsubsection{Navigation}

The core of the software is the target tracking. This module decides which targets to track and what observables to obtain. The camera processing module takes the camera data and converts the data into matrix format. This is then processed by the camera processing algorithms which perform star identification, planet centroiding, and landmark identification. It ultimately outputs the centroids of the identified targets. The centroids are processed by the recursive navigation module (Fig. 4), the attitude determination module, and the batch navigation module.

The image processing algorithm is shown in Fig. 5. There are two types of image processing required for the system. The first is to compute centroids of extended objects. The approach is to use Shen-Castan edge detection [11] to find the limb of a planetary image and to compute the centroids from curve fits to the limb. This permits partial disks, such as those due to phasing, to be used. Edge detection is shown in Fig. 6.

The UKF is able to achieve greater estimation performance than the Extended Kalman Filter (EKF) through the use of the unscented transformation 


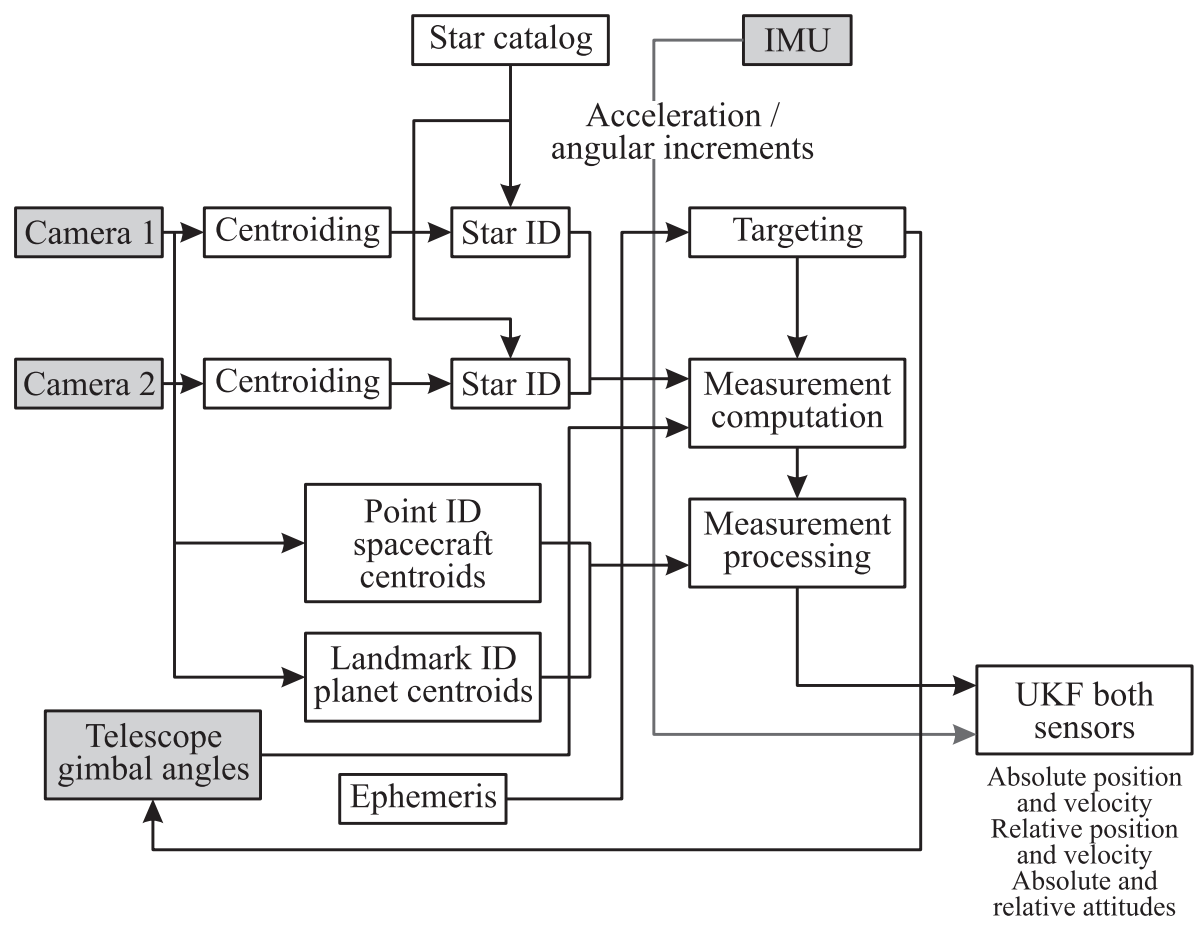

Figure 4 Recursive navigation

(UT). It is common to both the attitude determination and recursive navigation algorithms.

The UT allows the UKF to capture first- and second-order terms of the nonlinear system [12]. The state estimation algorithm employed is that given by van der Merwe [13] and the parameter estimation algorithm is that given by [12]. Unlike the EKF, the UKF does not require any derivatives or Jacobians of either the state equations or measurement equations. Instead of just propagating the state, the filter propagates the state and additional sigma points which are the states plus the square roots of rows or columns of the covariance matrix. Thus, the state and the state plus a standard deviation are propagated. This captures the uncertainty in the state. It is not necessary to numerically integrate the covariance matrix.

The filters use a numerical simulation to propagate the state. No specialized, linearized, or simplified simulations are needed. Thus, the algorithms can update the actual simulation used for flight operations. The only limitation is that only measurements that are available in telemetry can be used as measurements in the model. This means that some parameters will not be iden- 


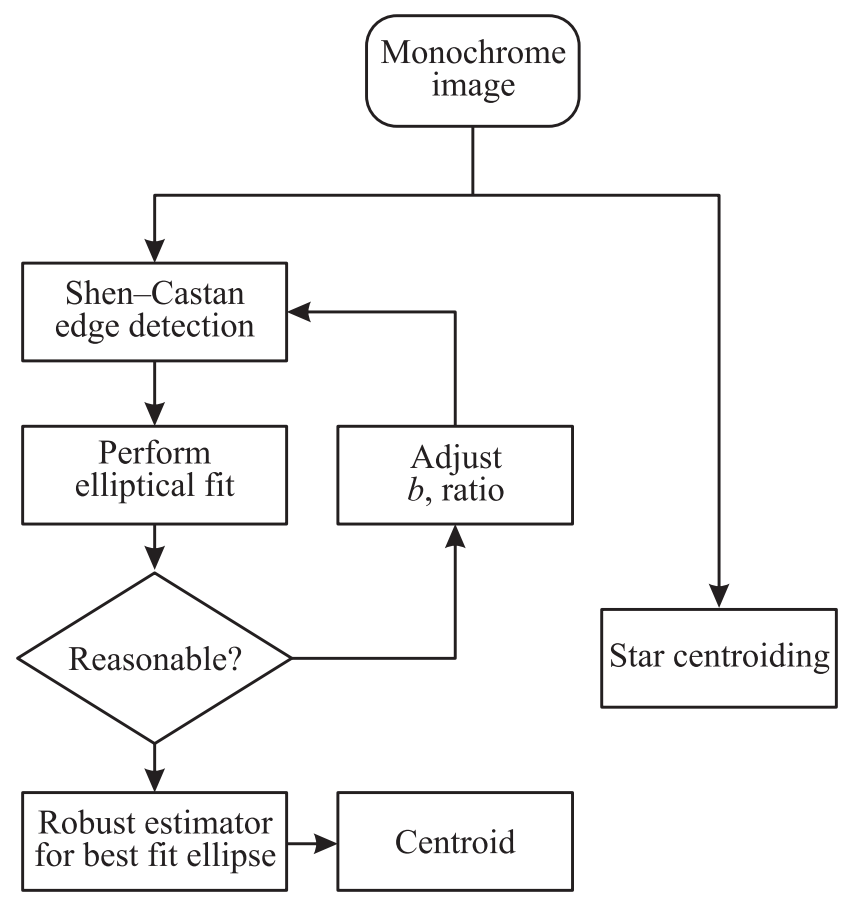

Figure 5 Centroiding of a partial lunar disk
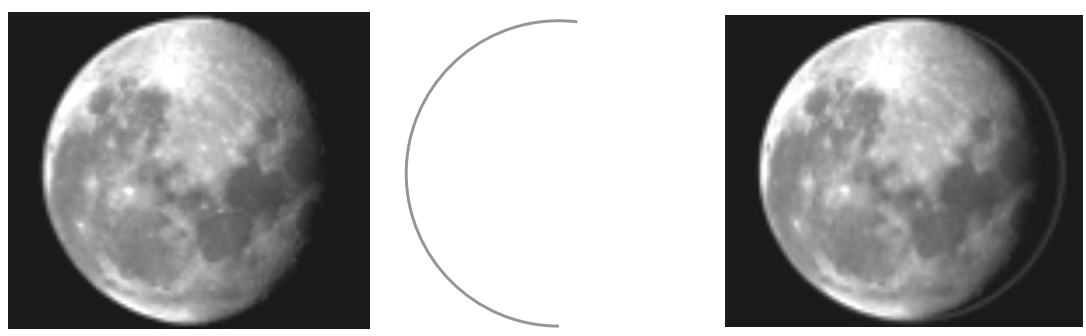

Figure 6 Moon centroiding. The detected edge is shown in the middle and its fit on the right 
tifiable. The visibility of parameters will depend on the values of the system states and the dynamical coupling between states. One issue that cannot be avoided with system identification is the need for there to be sufficient excitation of the system at a level to make parameter changes apparent in the measurements.

\subsubsection{Calibration}

The ICONS sensor is designed to be calibrated in space using a calibration cube mounted to the spacecraft. The camera looks at the corner of the cube which proves 108 known points for calibration. Each point has a different threedimensional (3D) coordinate. The center of the cube is also known.

It is necessary to calibrate the camera so that the position of a feature on the image plane can be correctly mapped to the direction in space of the actual physical feature. The ICONS sensor is designed to be calibrated in space by imaging a calibration cube mounted to the spacecraft. The camera looks at the corner of the cube and can see three faces of the cube. Each face carries nine squares each with four corners providing 108 features for calibration. Each feature is point-like and has a unique known $3 \mathrm{D}$ coordinate relative to the camera. The coordinate of the center of the cube is also known. The positions on the sensor of the 108 calibration features are then used to find an optimum set of calibration parameters.

Calibration parameters are grouped into two classes, extrinsic and intrinsic. Extrinsic parameters are those that involve the position and orientation of the camera, while intrinsic parameters involve the transformation of physical coordinates to pixel coordinates and takes into account the projection system employed by the lens system, distortions, center offset, and the like.

An attractive method used to find the optimum set of parameters is a two-step process. In the first step, lens distortion is ignored and a perspective projection is used which leads to a linearized model. The model can be approximately inverted using a Direct Linear Transformation (DLT). This approximate solution is used in step two as the starting point of a numerical optimization.

The position of a feature on the focal plane image is

$$
\vec{p}_{c}=\vec{R} \vec{p}_{w}+\vec{t}
$$

where $\vec{p}_{c}$ is the coordinate vector of the feature in the image frame of reference (that is, on the focal plane array); $\vec{p}_{w}$ is the coordinate of the feature in the spacecraft frame (i. e., relative to the camera); $\vec{R}$ is the matrix that transforms from the spacecraft frame to the image frame; and $\vec{t}$ is the offset between the center of the sensor and the center of the optics. The following extrinsic parameters must be estimated: 
- $\vec{R}$ - the $3 \times 3$ rotation matrix; and

$-\vec{t}$ - the $3 \times 1$ center offset vector.

The following intrinsic parameters must also be estimated:

- $f_{x}=f / s_{x}$ - the focal length (in units of pixel size, where $s_{x}$ and $s_{y}$ are the effective size of each pixel; and $f$ is the focal length);

$-\alpha=s_{x} / s_{y}$ - the aspect ratio;

- the image center coordinates, $\vec{o}_{x}$ and $\vec{o}_{y}$; and

- the radial distortion coefficients $k_{1}$ and $k_{2}$.

Given the 108 known points and the locations of their centroids in the focal plane, one can determine these parameters.

The location (pixel) of a feature is related to its coordinate (generally distorted by the optics) on the sensor by

$$
x_{d}=-\left(x_{\mathrm{im}}-o_{x}\right) s_{x} ; \quad y_{d}=-\left(y_{\mathrm{im}}-o_{y}\right) s_{y} .
$$

These coordinates are related to the actual physical location of the feature in space by the camera projection, typically

$$
x_{d}=-\frac{f}{s_{x}} \frac{x_{c}}{z_{c}} ; \quad y_{d}=-\frac{f}{s_{y}} \frac{y_{c}}{z_{c}}
$$

where $x_{c}, y_{c}$, and $z_{c}$ are the actual physical coordinates of the feature. Ideal, undistorted locations are related to the measured, distorted, and sensor coordinates by use of the radial distortion coefficients:

$$
x=x_{d}\left(1+k_{1} r_{d}^{2}+k_{2} r_{d}^{4}\right) ; \quad y=y_{d}\left(1+k_{1} r_{d}^{2}+k_{2} r_{d}^{4}\right)
$$

where $x$ and $y$ are the undistorted locations; $x_{d}$ and $y_{d}$ are the distorted locations; $r$ is the radial distance; and $k_{1}$ and $k_{2}$ are the distortion coefficients. The radial distance is

$$
r_{d}=x_{d}^{2}+y_{d}^{2}
$$

To model the radial distortions, that is, to go from undistorted to distorted coordinates, let combine the two equations in (4) to get

$$
x^{2}+y^{2}=r^{2}=r_{d}^{2}\left(1+k_{1} r_{d}^{2}+k_{2} r_{d}^{4}\right)^{2} .
$$

This results in the fifth-order equation:

$$
r=r_{d}\left(1+k_{1} r_{d}^{2}+k_{2} r_{d}^{4}\right) \text { or } k_{2} r_{d}^{5}+k_{1} r_{d}^{3}+r_{d}-r=0 .
$$

There is only one real solution for $r_{d}$. 
Table 1 Calibration results. The top set of parameters are estimated with downhill simplex

\begin{tabular}{ccc}
\hline Parameter & True & Estimate \\
\hline$R$ & {$\left[\begin{array}{lll}1.0 & 1.0 & 1.0\end{array}\right]$} & {$\left[\begin{array}{lll}1.0 & 1.0 & 1.0\end{array}\right]$} \\
$T$ & {$\left[\begin{array}{lll}0.0 & 0.0 & 0.0\end{array}\right]$} & {$\left[\begin{array}{lll}0.0 & 0.0 & 23.3\end{array}\right]$} \\
$f$ & {$\left[\begin{array}{ccc}10000.0 & 10000.0\end{array}\right]$} & {$\left[\begin{array}{lll}11070.2 & 11070.2\end{array}\right]$} \\
$k_{1}$ & $10^{-10}$ & 0.0 \\
$k_{2}$ & $10^{-14}$ & 0.0 \\
\hline$R$ & {$\left[\begin{array}{llll}1.0 & 1.0 & 1.0\end{array}\right]$} & {$\left[\begin{array}{lll}1.0 & 1.0 & 1.0\end{array}\right]$} \\
$T$ & {$\left[\begin{array}{lll}0.0 & 0.0 & 0.0\end{array}\right]$} & {$\left[\begin{array}{lll}0.0 & 0.0 & -0.0\end{array}\right]$} \\
$f$ & {$\left[\begin{array}{lll}10000.0 & 10000.0\end{array}\right]$} & {$\left[\begin{array}{lll}10000.0 & 10000.0\end{array}\right]$} \\
$k_{1}$ & $10^{-10}$ & $10^{-10}$ \\
$k_{2}$ & $10^{-14}$ & $10^{-14}$ \\
\hline
\end{tabular}

Equations (1)-(3) can be combined into a set of two nonlinear equations with the actual physical coordinates and measured coordinates as inputs and the intrinsic parameters, extrinsic parameters, rotation matrix, and translation center as outputs. The 108 pairs of equations can be solved.

The process is to first formulate them as a set of linear equations with the radial distortions ignored and solve them using the singular value decomposition. The resulting solution is iterated upon using downhill simplex. Calibration is done in background after the measurements are taken.

The results of the calibration are shown below without and with the downhill simplex to solve for the $k_{1}$ and $k_{2}$ coefficients. The computation for $R$ and the first two elements of $T$ are identical but without downhill simplex, the radial errors case, a large error in $T_{3}$ and the focal lengths. This test used a 30millimeter calibration cube with the camera $200 \mathrm{~mm}$ from the cube. Each square is 5 by $5 \mathrm{~mm}$. Table 1 gives the results of the calibration software.

\section{SIMULATION RESULTS}

Simulations using a model of the New Horizons spacecraft with the navigation system attached were conducted in VisualCommander (Fig. 7). The first two graphics show the summary page. A $3 \mathrm{D}$ view of the spacecraft and Pluto are in the left-hand display. The upper right-hand display shows the two camera field-of-view cones and the celestial sphere. The lower right-hand display shows the spacecraft position in the solar system. The last graphic shows the orbit determination page. The widgets on the right are for sending commands. The covariance diagonals are plotted on the left and the distance from Pluto just plotted below. The tables in the middle give the data of objects seen by the two 


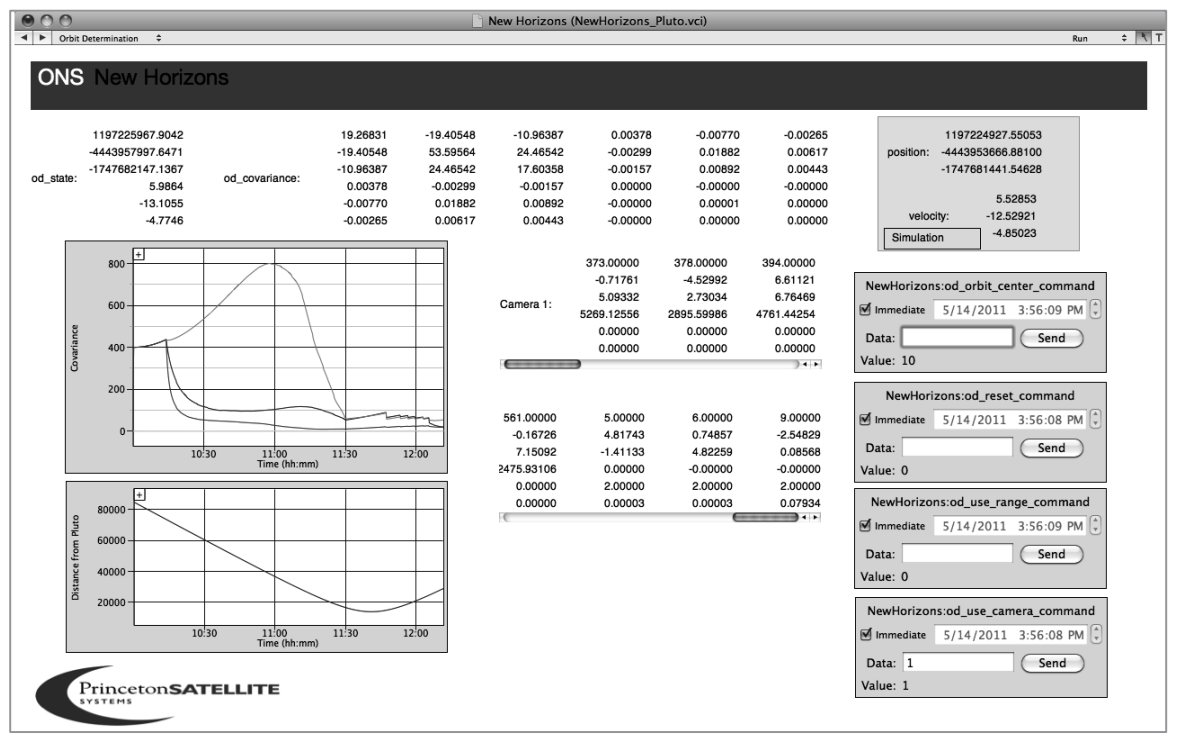

Figure 7 New Horizons Pluto flyby, data

cameras. SPICE IDs are used for the planets and the Hipparcos catalog IDs for the stars.

The attitude is allowed to converge before enabling the camera navigation measurements. One camera is pointed at Pluto and the second at Polaris. The aperture of the camera is $25 \mathrm{~mm}$ and the focal length is $50 \mathrm{~mm}$. This insures that both cameras see sufficient stars for attitude solutions throughout the flyby. The first figure shows the simulation as the spacecraft approaches Pluto. Pluto is visible in the graphics window and the tracking window shows the camera tracking Pluto. The second figure shows the tracking just after the flyby. The gimbals cannot move sufficiently fast to keep Pluto in the field of view. This causes a degradation of the navigation estimate. Once past Pluto, the camera sees multiple planets. It can use chordwidth and angles between planets for the length references. The covariance is shown in the third figure. Due to the geometry, the $y$-axis covariance increases. Near Pluto and beyond, it decreases to match the other two axes. The simulation tests absolute navigation. Relative navigation, i.e., Pluto centered, can also be enabled.

The control systems used in the simulations were developed at Princeton Satellite Systems. The system uses a PID (proportional-integral-derivative) controller which generates a torque demand which is converted into a pulse width demand by means of a linear program with slack variables. Both force and torque demands can be satisfied simultaneously. 


\section{CONCLUDING REMARKS}

This paper presents results for the optical navigation system. This system performs both orbit determination and attitude determination anywhere in the solar system. It can incorporate a wide variety of measurement sources. The navigation solutions are sufficient for orbit change maneuvers and other navigation functions. The next step in the development will be the manufacturing of a terrestrial version of the sensor followed by the development of a flight version and flight testing. Integrated communications provide a source of range and range rate measurements and timing information. A further improvement will be the addition of algorithms to optimally select targets based on their measurement uncertainty and the overall measurement geometry.

Currently, a CubeSat version of the hardware is being built. The CubeSat would not have articulated telescopes but would rely on the CubeSat maneuvering to point the telescopes. Precision reaction wheels, with a new type of motor, are under development and expected to produce much lower jitter than existing reaction wheels. A pair of CubeSats would allow testing of absolute navigation, relative navigation, and communications.

\section{ACKNOWLEDGMENTS}

This work was done under a NASA Contract No. NNX07CA46P. The authors would like to thank Dr. Owen Brown of JPL and Dr. Landis Markley of NASA/ GSFC for their contributions to this work.

\section{REFERENCES}

1. Battin, R.A. 2004. An introduction to the mathematics and methods of astrodynamics. AIAA. 755-56.

2. Bhaskaran, S., J. E. Reidel, and S. P. Synnott. 1996. Autonomous optical navigation for interplanetary missions. Proc. SPIE. 32-43.

3. Riedel, J. E., S. Bhaskaran, S. P. Synnott, S. D. Desai, W. E. Bollman, P. J. Dumont, C. A. Halsell, D. Han, B. M. Kennedy, G. W. Null, W. M. Owen, R. A. Werner, and B. G. Williams. 1997. Navigation for the New Millenium: Autonomous navigation for Deep Space 1. 12th Symposium (International) on Space Flight Dynamics Proceedings. Darmstadt.

4. Kubota, T., T. Hashimoto, S. SawaI, J. Kawaguchi, K. Ninomiya, M. Uo, and K. Baba. 2003. An autonomous navigation and guidance system for MUSES-C asteroid landing. Acta Astronautica 52(2-6):125-31. 
5. Kubota, T., S. Sawai, T. Hashimoto, and J. Kawaguchi. 2005. Robotics and autonomous technology for asteroid sample return mission. 12th Conference (International) on Advanced Robotics Proceedings. 31-38.

6. Foing, B. H., D. Heather, M. Almeida, G. Racca, and A. Marini. 2001. The ESA SMART-1 mission to the Moon - science and technology objectives. National Space Society's 20th Annual Space Development Conference (International) Proceedings.

7. Polle, B. 2006. Low thrust missions guidance and navigation: The Smart-1 OBAN experiment results and perspectives. 3rd Workshop (International) on Astrodynamics Tools and Techniques.

8. Polle, B., B. Frapard, T. Voirin, J. Gil-Fernandez, E. Milic, M. Graziano, R. Panzeca, J. Rebordao, B. Correia, M. Proenca, J. Dinis, P. Mortena, and P. Dyarte. 2003. Vision based navigation for interplanetary exploration opportunity for AURORA. Astronautical Congress (International) Proceedings.

9. Owen, W. M., Jr., and T. C. Wang. 2001. NEAR optical navigation at Eros. AAS 01-376.

10. Riedel, J. E., T. C. Wang, R. Werner, A. Vaughan, D. Myers, N. Mastrodemos, G. Huntington, C. Grasso, R. Gaskell, and D. Bayard. 2008. Configuring the deep impact AutoNav System for Lunar, Comet and Mars landing. AIAA/AAS Astrodynamics Specialist Conference and Exhibit. AIAA. @articl

11. Shen, J., and S. Castan. 1992. An optimal linear operator for step edge detection. CVGIP: Graphical Models Image Proc. 54(2):112-33

12. VanDyke, M. C., J. L. Schwartz, and C. D. Hall. 2004. Unscented Kalman filtering for spacecraft attitude state and parameter estimation. American Astronautical Society Proceedings. AAS-0115.

13. Van der Merwe, R., and E. A. Wan. 2001. The square-root unscented Kalman filter for state and parameter-estimation. 2001 IEEE Conference (International) on Acoustics, Speech, and Signal Processing Proceedings (ICASSP '01). 6:346164 . 